\title{
Adherence of Community Pharmacies in Riyadh, Saudi Arabia, to Optimal Conditions for Keeping and Selling Good-Quality Medicines
}

\author{
Hani M. J. Khojah ${ }^{1,2 *}$, Henrik Pallos ${ }^{3}$, Hirohito Tsuboi ${ }^{1}$, Naoko Yoshida ${ }^{1}$, Hisham S. Abou-Auda ${ }^{4}$, \\ Kazuko Kimura ${ }^{1}$ \\ ${ }^{1}$ Department of Drug Management and Policy, Graduate School of Natural Science and Technology, Kanazawa University, Kana- \\ zawa, Japan; ${ }^{2}$ Department of Clinical and Hospital Pharmacy, College of Pharmacy, Taibah University, Madinah, KSA; ${ }^{3}$ Department \\ of International Pharmaceutical Sciences, Graduate School of Natural Science and Technology, Kanazawa University, Kanazawa, \\ Japan; ${ }^{4}$ Department of Clinical Pharmacy, College of Pharmacy, King Saud University, Riyadh, KSA. \\ Email: "hani_khojah@yahoo.com
}

Received June $5^{\text {th }}, 2013$; revised July $8^{\text {th }}, 2013$; accepted July $31^{\text {st }}, 2013$

Copyright (C) 2013 Hani M. J. Khojah et al. This is an open access article distributed under the Creative Commons Attribution License, which permits unrestricted use, distribution, and reproduction in any medium, provided the original work is properly cited.

\begin{abstract}
Exposure to excessive temperature during distribution or storage has unfavorable consequences on the quality of medicines, particularly in hot climates. This could be one of the possible reasons behind the existence of substandard amoxicillin in community pharmacies in Riyadh, Saudi Arabia. This study explored the conditions under which medicines were kept in a random sample of 181 community pharmacies in Riyadh. The pharmacist in charge in each pharmacy was interviewed and our observations about the quality of storage were recorded. The inspection revealed that in $9 \%$ of the sample the readings of the existing room thermometers were $>25^{\circ} \mathrm{C}$, and that $13 \%$ of the sample lacked thermometers. Also in $33 \%$ of the sample the readings of the refrigerator thermometers were outside the accepted range, and $7 \%$ of the sample lacked refrigerator thermometers. About $15 \%$ of pharmacists were not informed about the local regulations of community pharmacy practice, neither before nor after taking up their current positions. Surprisingly, incorrect answers to simple questions about the system were frequently given by the informed pharmacists. Certain aspects of substandard storage conditions existed, in varying degrees, in significant percentages of pharmacies regardless of the pharmacists' qualifications, experience, or awareness about the local regulations of community pharmacy practice. Stricter monitoring by the authorities regarding the adherence of community pharmacies to optimal conditions of storing medicines is recommended. Continuing education of community pharmacists must also be improved.
\end{abstract}

Keywords: Quality of Medicines; Substandard Medicine; Saudi Arabia; Community Pharmacy; Quality of Pharmacies

\section{Introduction}

The quality of medicine is a global issue due to the ever increasing prevalence of counterfeit medicines worldwide [1,2]. Substandard medicines produced by legitimate manufacturers represent another threat as they may not contain the correct amount of active ingredients or may have manufacturing defects that alter their efficacy or make them dangerous for use $[3,4]$. On the other hand, even good quality medicines could be risky if they degrade due to poor storage or distribution conditions $[5$, 6].

This study is based on the results of a previous study

${ }^{*}$ Corresponding author. which is submitted for publication [7]. That study investigated the quality of amoxicillin capsules and tablets sold in community pharmacies in Riyadh, Saudi Arabia. It was conducted during a very hot season and amoxicillin was selected as an indicator of the quality of medicines in those pharmacies. The study reported substandard amoxicillin products in $13 \%$ of the randomly selected pharmacies. Although all samples were found to be authentic and the amount of the active ingredient was not dramatically low, it was below the pharmacopoeial limits with the lowest amount being 80.7\%. Manufacturing defects could not be ruled out but it was also suggested that degradation may have occurred to some samples due to poor storage or distribution conditions. 
That was supported by the finding that certain samples passed the quality test while other samples from the same batch, but purchased from other pharmacies, failed it.

Most medicines that can be kept on shelves must not be exposed to temperatures exceeding $25^{\circ} \mathrm{C}$. Excessive heat plays a major role in the degradation of various medicines $[8,9]$. Similarly, temperature-controlled medicines must be handled carefully to avoid decomposition or degradation caused by excessive heat [10].

The operational regulations of the system for private pharmaceutical facilities and products in Saudi Arabia contain several requirements for good storage and distribution of medicines [11]. According to this system, community pharmacies must have good air-conditioning systems that keep the temperature at $\leq 25^{\circ} \mathrm{C}$ and a thermometer for monitoring the temperature. Pharmacies must also have refrigerators with thermometers and medicines must be kept away from sunlight. Floors and paints must be of materials that can be easily washed and cleaned. In addition, drug storage facilities must be equipped with automatic temperature recording systems that keep records for at least one year.

However, the criteria and quality of inspection and monitoring of the community pharmacies by the regulatory authorities may not be efficient. For example, one can easily buy prescription-only medicines, such as antibiotics, without a prescription despite the strict regulations [12].

Our current study explored the extent to which community pharmacies in Riyadh complied with the local regulations for keeping medicines until they are sold. We also explored the opinions of community pharmacists about the quality of medicines and tested their knowledge about the regulations.

\section{Materials and Methods}

\subsection{Ethical Issues}

This study was approved by the Ethics Committee of Kanazawa University and the Saudi Food and Drug Authority (SFDA), and was conducted between November 2 and November 23, 2012.

\subsection{Selection of Pharmacies}

A list of all registered community pharmacies in Riyadh was obtained from the Saudi Ministry of Health $(\mathrm{MOH})$ in August 2012 (1531 pharmacies). The calculated sample size of pharmacies was 181 according to the following formula:

$$
n=z^{2} N(1-p) /\left(z^{2} p(1-p)+\xi^{2} p^{2}(N-1)\right)
$$

where $\boldsymbol{n}$ is the required sample size; $\boldsymbol{z}$ is the reliability coefficient (equals $1.96 \%$ at $95 \%$ confidence level); $N$ is the population size; $\boldsymbol{p}$ is the probability of favorable outcome (set as 0.5 ); and $\xi$ is set as 0.2 for the purpose of this study.

After all pharmacies were coded, the list was scrambled and $181+45$ pharmacies were randomly selected from the list with MS Excel 2010 (Microsoft Co., USA). The additional 45 pharmacies were used as a reserve for an estimated dropout rate of $25 \%$ that may occur when a pharmacy is closed on the second visit, the pharmacy is out of business, or the pharmacy refuses to cooperate in the survey.

\subsection{Study Materials}

The survey was conducted using a structured interview with the pharmacist in charge in each pharmacy, using 3 forms that were filled in by the interviewers. The first form was a questionnaire while the second and third forms were used for inspection and observation purposes, respectively. Validity of the method was assessed on a sample of 33 pharmacies, not included in the study sample, and the Pearson correlation coefficient for internal reliability was 0.82 .

\subsection{The Interviewers}

Thirty-four, fourth-year pharmacy students from King Saud University in Riyadh conducted the interviews after having attended a workshop followed by field training for a period of one week accompanied by the chief investigator. In-field follow-up and necessary support were provided by the chief investigator and one of the co-investigators.

\section{Results}

The final sample included 139 chain and 42 independent pharmacies, where a chain pharmacy belongs to a group of $>3$ pharmacies [13]. Twenty-one pharmacies were replaced because they were either closed on the second visit, out of business, or non-cooperative. The pharmacists in charge were all non-Saudi males and the summary of their background characteristics is shown in Table 1.

About $15 \%$ of the pharmacists said that they had not been informed about the system of community pharmacy practice in Saudi Arabia. Surprisingly, a significant percentage of pharmacists who knew the system gave wrong answers to simple questions related to the system. Table 2 summarizes the pharmacists' answers to such questions.

All pharmacists reported that they always receive the supply of medicines either directly from an official distributor or from a central store that belongs to the owner, or both. However, one independent pharmacy reported purchasing some medicines from a subagent. Nearly $56 \%$ 
Table 1. Background characteristics of pharmacists.

\begin{tabular}{lll}
\hline Characteristics $^{\mathrm{a}}$ & Value & \\
\hline & Mean (years) & Range \\
Age & 32 & $23-55$ \\
Total experience in community & 8.2 & $1-28$ \\
pharmacies & & \\
Experience in community & 5.3 & $0.25-25$ \\
pharmacies in Saudi Arabia & Frequency & $\%$ \\
& $(n=181)$ & \\
Qualification & & \\
B.Sc & 174 & 96.1 \\
Pharm. D & 4 & 2.2 \\
M.Sc & 2 & 1.1 \\
Ph.D & 1 & 0.6 \\
Nationality & & \\
Egypt & 150 & 82.9 \\
Yemen & 7 & 3.8 \\
Jordan & 6 & 3.3 \\
India & 6 & 3.3 \\
Sudan & 514.4 \\
Syria & 5 & 2.8 \\
Palestine & 3 & 1.7 \\
Pakistan & & 1.7 \\
Position & & 0.5 \\
Manager & & \\
Staff & & \\
\hline
\end{tabular}

${ }^{\mathrm{a} A l l}$ were males.

of the pharmacists reported that they sometimes rejected some supplies of medicines because the temperature of the shipments may have exceeded $25^{\circ} \mathrm{C}$ or because the packaging of the medicines was not intact upon receipt. When asked about the official distributors' facilities, $16 \%$ of the pharmacists said that the distributors' stores were substandard and $22 \%$ believed that the vehicles that were delivering the supply to the pharmacies were not suitable. In addition, $8 \%$ reported that they found some counterfeit products in their pharmacies and $46 \%$ reported that they had sometimes been contacted by illegal sellers offering known medicine brands at low prices. A significant percentage of the pharmacists reported that they found physicochemical changes in some of the medicines in their pharmacies: $16 \%$ noticed discoloration of some liquid medicines while $18.8 \%$ noticed wetting or solidification of powdered medicines. Sixty-seven percent of the pharmacists reported that they received complaints from their clients about subtherapeutic effects of some medicines. Only $64 \%$ said that they received memos from the drug regulatory authorities warning them of certain counterfeit or substandard products. Table 3 summarizes the pharmacists' opinions about the compliance of their pharmacies as well as their drug distributors with the local regulations.

In all pharmacies, excess medicines were stored in a small room inside each pharmacy. The inspection revealed serious problems regarding the temperature control in the pharmacies and in their refrigerators. Generally, the degree of cleanliness and neatness of pharmacies was considered acceptable since no serious breach was found. A summary of the recordings of inspections and observations made in all pharmacies is presented in Table 4.

Various scoring systems were used in different studies concerned with the quality of pharmacies for a variety of statistical analyses [14,15]. In our study, however, when

Table 2. Pharmacist knowledge about the local regulations of community pharmacy practice.

\begin{tabular}{ll}
\hline Aspects & $\begin{array}{l}\text { Frequency, } \\
n=181(\%)\end{array}$ \\
\hline $\begin{array}{l}\text { Were you informed about the community } \\
\text { pharmacy regulations in Saudi Arabia? }\end{array}$ & \\
Yes & $153(84.5)$ \\
No & $28(15.4)$
\end{tabular}

Are you requested to complete a certain number of continuing education hours every year?
Yes, 60 hours $^{\mathrm{a}}$
$49(27.0)$
Yes, incorrect number of hours
$81(44.8)$
Not required
$32(17.7)$
Don't know
19 (10.5)

Is it allowed to give free samples of over-the-counter medicines to clients?

$\begin{array}{ll}\text { Yes } & 21(11.6) \\ \mathrm{No}^{\mathrm{a}} & 155(85.6) \\ \text { Unsure } & 5(2.8)\end{array}$

Except antibiotics, is it allowed to sell medicines by individual strips?

$\begin{array}{ll}\text { Yes }^{\mathrm{a}} & 95(52.4) \\ \text { No } & 81(44.8) \\ \text { Unsure } & 5(2.8)\end{array}$

Correct answers about some medicines that can be sold without a prescription
Mild cough preparations
$157(86.7)$
Topical disinfectants
$143(79.0)$
Multivitamins
$158(87.3)$
Topical burn preparations
$139(76.8)$
Mild analgesics
$167(92.3)$

Correct answers about some medicines that cannot be sold without a prescription

$\begin{array}{ll}\text { Antihypertensive drugs } & 172(95.0) \\ \text { Mild oral antibiotics } & 138(76.2) \\ \text { Strong oral antibiotics } & 169(93.4) \\ \text { Antidiabetic drugs } & 166(91.7)\end{array}$

${ }^{\mathrm{a}}$ The correct answer. 
Table 3. Pharmacists' opinions on the adherence of their pharmacies and distributors to the regulations.

\begin{tabular}{|c|c|}
\hline Aspects & Frequency, $n=181(\%)$ \\
\hline \multicolumn{2}{|l|}{ How often is the pharmacy temperature kept at $\leq 25^{\circ} \mathrm{C}$ during working hours in hot seasons? } \\
\hline $100 \%$ & $146(80.7)$ \\
\hline $91 \%-99 \%$ & $12(6.6)$ \\
\hline $81 \%-90 \%$ & $12(6.6)$ \\
\hline$\leq 80 \%$ & $9(5.0)$ \\
\hline \multicolumn{2}{|l|}{ How often is the pharmacy temperature kept at $\leq 25^{\circ} \mathrm{C}$ after working hours in hot seasons? } \\
\hline $100 \%$ & $119(65.7)$ \\
\hline $91 \%-99 \%$ & $15(8.3)$ \\
\hline $81 \%-90 \%$ & $21(11.6)$ \\
\hline$\leq 80 \%$ & $19(10.5)$ \\
\hline \multicolumn{2}{|l|}{ How often is the supply delivered to the pharmacy at a temperature of $\leq 25^{\circ} \mathrm{C}$ in hot seasons? } \\
\hline $100 \%$ & $102(56.3)$ \\
\hline $91 \%-99 \%$ & $5(2.8)$ \\
\hline $81 \%-90 \%$ & $11(6.1)$ \\
\hline$\leq 80 \%$ & $21(11.6)$ \\
\hline Unsure & $42(23.2)$ \\
\hline \multicolumn{2}{|l|}{ How often is the supply received in intact packaging? } \\
\hline $100 \%$ & $109(60.2)$ \\
\hline $91 \%-99 \%$ & $41(22.7)$ \\
\hline $81 \%-90 \%$ & $14(7.7)$ \\
\hline$\leq 80 \%$ & $8(4.4)$ \\
\hline Unsure & $9(5.0)$ \\
\hline $100 \%$ & $71(39.2)$ \\
\hline $91 \%-99 \%$ & $20(11.1)$ \\
\hline $81 \%-90 \%$ & $36(19.9)$ \\
\hline$\leq 80 \%$ & $29(16.0)$ \\
\hline Unsure & $25(13.8)$ \\
\hline \multicolumn{2}{|l|}{ To what extent do you believe that Riyadh pharmacies adhere to optimal storage conditions? } \\
\hline $100 \%$ & $45(24.8)$ \\
\hline $91 \%-99 \%$ & $21(11.6)$ \\
\hline $81 \%-90 \%$ & $38(21.0)$ \\
\hline$\leq 80 \%$ & $46(25.4)$ \\
\hline Unsure & $31(17.2)$ \\
\hline \multicolumn{2}{|l|}{ How often is the pharmacy inspected by the pharmacy regulatory authorities? } \\
\hline Every 1 - 6 months & $129(71.3)$ \\
\hline Every 7 - 12 months & $19(10.5)$ \\
\hline Every 1 - 2 years & $3(1.6)$ \\
\hline Unsure & $30(16.6)$ \\
\hline The pharmacist noticed broken capsules or tablets inside packaged products in the pharmacy & $53(29.3)$ \\
\hline The pharmacist noticed discoloration of some liquid medicines in the pharmacy & $29(16.0)$ \\
\hline The pharmacist noticed wetting or solidification of powdered medicines in the pharmacy & $34(18.8)$ \\
\hline Electricity blackout occurred during summer and pharmacy temperature raised above $25^{\circ} \mathrm{C}$ & $71(39.2)^{\mathrm{a}}$ \\
\hline
\end{tabular}

${ }^{\mathrm{a}}$ Infrequent and lasted for 15 - 180 minutes. 
Table 4. Pharmacy inspection results and observations.

\begin{tabular}{|c|c|}
\hline Elements & Frequency, $n=181(\%)$ \\
\hline Availability of an alternative power supply that covers the air-conditioning and refrigerator & $10(5.5)$ \\
\hline Availability of an additional air-conditioner & $147(81.2)$ \\
\hline Availability of a thermometer to measure pharmacy temperature & $157(86.7)$ \\
\hline Pharmacy thermometer reading was $\leq 25^{\circ} \mathrm{C}^{\mathrm{a}}$ & $143(91.1), n=157$ \\
\hline Availability of a refrigerator & $179(98.9)$ \\
\hline Availability of a thermometer in the refrigerator & $167(93.3), n=179$ \\
\hline Refrigerator thermometer reading was $3^{\circ} \mathrm{C}-8^{\circ} \mathrm{C}^{\mathrm{b}}$ & $111(66.5), n=167$ \\
\hline Walls behind medicine shelves were not struck by direct sunlight from the outside & $136(75.1)$ \\
\hline Walls behind medicine shelves were not hot or warm & $173(95.6)$ \\
\hline All medicines were not exposed to direct sunlight & $160(88.4)$ \\
\hline Availability of at least 1 comprehensive drug information reference & $37(20.4)$ \\
\hline Availability of a copy of the local regulations for community pharmacy practice & $55(30.4)$ \\
\hline Free medicine samples were not seen & $176(97.2)$ \\
\hline Medicine advertisement was not seen & $153(84.5)$ \\
\hline There was a sign that states that prescription drugs cannot be sold without a prescription & $158(87.3)$ \\
\hline Shelves were clean ${ }^{\mathrm{c}}$ & $128(70.7)$ \\
\hline Walls were clean ${ }^{\mathrm{c}}$ & $135(74.6)$ \\
\hline Floor was clean ${ }^{\mathrm{c}}$ & $142(78.5)$ \\
\hline Floor was smooth/washable & $181(100)$ \\
\hline $\begin{array}{l}\text { Pharmacy was neatly organized (medicines were organized in shelves, shelves were organized in space, } \\
\text { and pharmacy was not overcrowded with products) }\end{array}$ & $147(81.2)$ \\
\hline
\end{tabular}

${ }^{a}$ Readings as high as $30^{\circ} \mathrm{C}$ were observed in some pharmacies. ${ }^{b}$ Readings as low as $-10^{\circ} \mathrm{C}$ and as high as $20^{\circ} \mathrm{C}$ were observed in some pharmacies. ${ }^{\mathrm{c}} \mathrm{Free}$ of dust, dirt, insects, or spider webs.

scores were added to key observations, no significant differences were found between the means of various independent factors, for example, chain vs. independent pharmacies, managers $v s$. staff, pharmacist informed about the regulations in Saudi Arabia vs. those not informed, and pharmacists aware of the continuing education requirements vs. those who were unaware. The analysis of variance is not possible within nationality and qualification groups because the number of candidates in some of these groups was very small compared to others. Also, no correlation is found between the scores and scale measures such as age and experience.

\section{Discussion}

Our findings suggest that there were some deficiencies in the storage of medicines in community pharmacies in Riyadh and probably in the delivery vehicles, especially during hot seasons. This may, at least partly, explain the existence of substandard levels of amoxicillin in Riyadh pharmacies [7]. Even basic thermometers were not available in about $13 \%$ of pharmacies, and in about $9 \%$ of the pharmacies equipped with a thermometer, the reading exceeded the $25^{\circ} \mathrm{C}$ threshold. About $19 \%$ of pharmacies lacked a spare air-conditioner, making the quality of medicines questionable if the only available air-conditioner fails to operate optimally during summer. What is worse, some pharmacists reported that the air-conditioners may not have been kept running after working hours in hot seasons.

In addition, about $7 \%$ of refrigerators lacked thermometers, and in about $33 \%$ of refrigerators, the temperatures were outside the accepted range. In about $25 \%$ of the pharmacies, some walls behind medicine shelves were struck by direct sunlight from the outer side, and in about $4 \%$ of those pharmacies the walls felt warm.

The local regulations must be updated accordingly to ensure the best storage and distribution conditions for the 
medicines. Such detailed conditions and specifications may be obtained from the WHO [16,17]. The existence of an alternative power supply that covers the air-conditioning and refrigerators might be necessary in Riyadh although electricity blackouts are infrequent. However, if the durations of blackouts are enough to raise the temperature inside the pharmacy or the refrigerator above the allowed limits, then at least the refrigerator and one airconditioning unit must be linked to an alternative power supply in each pharmacy.

A significant percentage of pharmacists were unaware of the basic regulations relating to community pharmacy practice. The striking issue is that having knowledge about the regulations had no impact on the quality of storage in the pharmacies. This may explain the absence of any correlation between age or experience and the general score achieved. This also explains the lack of any significant differences between various grouping factors relative to the mean scores.

Stricter, periodic monitoring and inspection by the authorities are highly recommended. We suggest that all pharmacy owners must add spare air-conditioners as a prerequisite for licensing the pharmacies. Each pharmacy must be equipped with a room thermometer and a refrigerator thermometer that keep a record of temperature variation during the day. In addition, the pharmacy location must be in a position where its walls are not struck by direct sunlight from the outer side, or at least such walls must be adequately insulated. Medicine shelves must not be placed near the entrance or any location exposed to direct sunlight. Distributors' storage and delivery facilities must also be strictly monitored.

Finally, the continuing education program for community pharmacists must be closely monitored and supervised by the authorities. We also recommend that passing an annual test about good pharmacy practice (GPP) should be a prerequisite for renewing the pharmacist license.

\section{Conclusions}

The quality of on-shelf medicines sold in community pharmacies in Riyadh may be questionable during hot seasons. Meanwhile, refrigerated medicines may not meet the quality standards throughout the entire storage time. More assertive measures and stricter monitoring of the adherence of the community pharmacies to good practice and good storage regulations are highly recommended. Community pharmacists' continuing education and knowledge about the practice regulations must be improved.

\section{Acknowledgements}

The authors would like to thank the SFDA and the Saudi
$\mathrm{MOH}$ for providing the necessary support and information about the pharmacies.

\section{Authors' Contribution}

Research protocol development and manuscript revision: all authors. Field work: HMJK and HSA.

\section{REFERENCES}

[1] K. Dégardina, Y. Roggoaand and P. Margot, "Understanding and Fighting the Medicine Counterfeit Market," In Press for Journal of Pharmaceutical and Biomedical Analysis, 2013. doi:10.1016/j.jpba.2013.01.009

[2] T. K. Mackey and B. A. liang, "The Global Counterfeit Drug Trade: Patient Safety and Public Health Risks," Journal of Pharmaceutical Sciences, Vol. 100, No. 11, 2011, pp. 4571-4579. doi:10.1002/jps.22679

[3] J. M. Caudron, N. Ford, M. Henkens, C. Macé, R. Kiddle-Monroeand and J. Pine, "Substandard Medicines in Resource-Poor Settings: A Problem That Can No Longer be Ignored," Tropical Medicine and International Health, Vol. 13, No. 8, 2008, pp. 1062-1072. doi:10.1111/j.1365-3156.2008.02106.x

[4] O. Shakoor, R. B. Taylor and R. H. Behrens, "Assessment of the Incidence of Substandard Drugs in Developing Countries," Tropical Medicine and International Health, Vol. 2, No. 9, 1997, pp. 839-845. doi:10.1046/j.1365-3156.1997.d01-403.x

[5] F. M. Fernandez, D. Hostetler, K. Powell, H. Kaur, M. D. Green, D. C. Mildenhall and P. N. Newton, "Poor Quality Drugs: Grand Challenges in High Throughput Detection, Countrywide Sampling, and Forensics in Developing Countries," Analyst, Vol. 136, No. 15, 2011, pp. 30733082. doi:10.1039/c0an00627k

[6] P. N. Newton, A. A. Amin, C. Bird, P. Passmore, G. Dukes, G. Tomson, B. Simons, R. Bate, P. J. Guerin and N. J. White, "The Primacy of Public Health Considerations in Defining Poor Quality Medicines," PLoS Medicine, Vol. 8, No. 12, 2011, Article No: e1001139. doi:10.1371/journal.pmed.1001139

[7] H. M. J. Khojah, H. Pallos, N. Yoshida, M. Akazawa, H. Tsuboi and K. Kimura, "The Quality of Medicines in Community Pharmacies in Riyadh, Saudi Arabia: A Lot Quality Assurance Sampling (LQAS)-Based Survey," unpublished, under reviewing.

[8] B. Crichton, "Keep in a Cool Place: Exposure of Medicines to High Temperatures in General Practice during a British Heat wave," Journal of the Royal Society of Medicine, Vol. 97, No. 7, 2004, pp. 328-329. doi: $10.1258 /$ jrsm.97.7.328

[9] K. K. Naidoo, P. Nompuku, S. N. Mkalali, K. Shabangu, L. Nkabinde and V. Singh, "Post-Marketing Stability Surveillance: Amoxicillin," South African Family Practice, Vol. 48, No. 6, 2006, p. 14.

[10] R. Ziance, C. Chandler and R. H. Bishara, "Integration of Temperature-Controlled Requirements into Pharmacy Practice," Journal of the American Pharmacists Associa- 
tion, Vol. 49, No. 3, 2003, pp. 61-67.

doi:10.1331/JAPhA.2009.08140

[11] Saudi Food and Drug Authority (SFDA), "Operational Regulations of the System for Private Pharmaceutical Facilities and Products," in Arabic, accessed 2013 May 10. http://sfda.gov.sa/ar/drug/drug_reg/DocLib/Executiverole sforInstitutionsandPharmaceuticalProductslaw.pdf

[12] A. A. B. Abdulhak, M. A. Altannir, M. A. Almansor, M. S. Almohaya, A. S. Onazi, M. A. Marei, O. F. Aldossary, S. A. Obeidat, M. A. Obeidat, M. S. Riaz and I. M. Tleyjeh, "Non Prescribed Sale of Antibiotics in Riyadh, Saudi Arabia: A Cross Sectional Study," BMC Public Health, Vol. 11, 2011, p. 538. doi:10.1186/1471-2458-11-538

[13] T. A. O. Najjar, "A Survey on Community Pharmacies in Riyadh, Saudi Arabia," Saudi Pharmaceutical Journal, Vol. 9, No. 2, 2001, pp. 113-118.

[14] Z. A. Butt, A. H. Gilani, D. Nanan, A. L. Sheikh and F.
White, "Quality of Pharmacies in Pakistan: A Cross-Sectional Survey," International Journal for Quality in Health Care, Vol. 17, No. 4, 2005, pp. 307-313. doi:10.1093/intqhe/mzi049

[15] L. Syhakhang, B. Stenson, R. Wahlström and G. Tomson, "The Quality of Public and Private Pharmacy Practices," European Journal of Clinical Pharmacology, Vol. 57, No. 3, 2001, pp. 221-227. doi:10.1007/s002280100295

[16] World Health Organization, "WHO Expert Committee on Specifications for Pharmaceutical Preparations," ThirtySeventh Report, World Health Organization, 2004, pp. 125-136, Accessed 2013 May 10.

http://whqlibdoc.who.int/trs/who_trs_908.pdf

[17] World Health Organization, "WHO Expert Committee on Specifications for Pharmaceutical Preparations," Fortieth Report, World Health Organization, 2006, pp. 179-202. Accessed 2013 May 10.

http://whqlibdoc.who.int/trs/who_trs_937_eng.pdf 